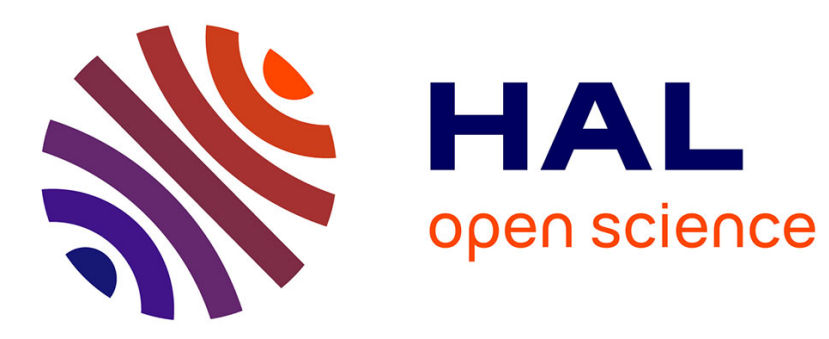

\title{
Internal Model Control and Max-Algebra: Controller Design
}

Jean-Louis Boimond, Jean-Louis Ferrier

\section{To cite this version:}

Jean-Louis Boimond, Jean-Louis Ferrier. Internal Model Control and Max-Algebra: Controller Design. IEEE Transactions on Automatic Control, 1996, 41 (3), pp.457-461. hal-00843583

\section{HAL Id: hal-00843583 \\ https://hal.science/hal-00843583}

Submitted on 15 Jul 2013

HAL is a multi-disciplinary open access archive for the deposit and dissemination of scientific research documents, whether they are published or not. The documents may come from teaching and research institutions in France or abroad, or from public or private research centers.
L'archive ouverte pluridisciplinaire HAL, est destinée au dépôt et à la diffusion de documents scientifiques de niveau recherche, publiés ou non, émanant des établissements d'enseignement et de recherche français ou étrangers, des laboratoires publics ou privés. 


\section{Internal Model Contmol and Max-Algebra: Controller Design}

Jean-Louis Boimond and Jean-Louis Ferrier

Abstroct-This noie proposes sn iniernal model control for linear discrele-syent systems over max-algebra. We shail coneenirate on the controlier block of this centrol sructure.

\section{INTRODLCIION}

We are interested in the control of discrete-event systems (DES's) which can be modeled by deterministic timed-event graphs (TEG's). It is well known that this particular class of graphs (a subset of the more general class of Petri nets [3], [4] can be linearly described in max-algebra. Moreover, this algebra allows interesting concepts from conventional linear system theory 10 motivate the study of DES's [2]. [3].

This paper deals with the following control problem: 10 fire at the latest date the process input so that the firing dates of the process output occur at the latest before the desired ones (described by the reference input). An sttractive solution to this problem exists and is given in $[2$, Section $X]$ and $[3$, Section 5.6$]$ in the particular case where all the values of the desired process output are available and the model is exactly known. The control system offers a strong analogy with the adjoint system of conventional optimal control theory. Our motivation is to consider here some more general assumptions:

- Only the past values of the desired process output are available which prevents us from applying the previous solution:

- Mismatch between the process and its model can exist which leads us to consider a feedback control structure rather than the open-loop control structure used in the previous solutiun. We choose the intemal model control (IMC) structure used in conventional control thenry because it is recognized as very useful to take into account imperfect modeling.

This note is organized as follows. Section II deals briefly with the linear model representation in max-alecbra. Basic IMC structure is introduced in Seetion III; the stability guarantee, in case of important mismatch between the process and its model, is not addressed hers: Besides the model, this control structure includes a controller. Its design in mas-algebra is described in Section IV. The unavailability of all the future firing dates of the controller input prevents us from having the exact solution to the previous control problem. However, in the proposed method we try to control the model in such a way that the furure firing dates of the model output occur as close as possible to the ones defined by the predicted controller input. This input is the reference input modified to take into account mismatch between the process and its model With such a procedure, the difference between the process outpu and the reference inpur depends on the prediction quality of the modified reference input. Specific problems of prediction are not discussed in this note; we only define the instants when the predicted modified reference input must be available. Section $\mathrm{V}$ applies the IMC design to a shon example.

Manuscript received Uetober 11, 1994; revised July 5, 1995 and September 12. 1995 .

The authors are with the Leboratoire d'Ingenierie des Srstemes Auromatists (LSA), Notre Dame du Lac, 490000 Angers, France.

Publisher lien Identifier S $0018-9286(96) 02258$ -

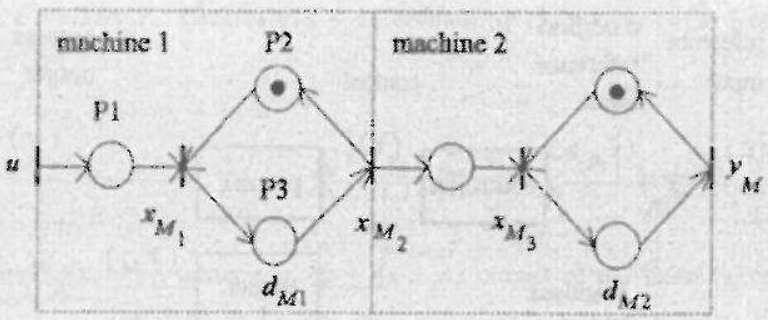

Fig. 1. A THG.

\section{LINBAR ALGERRAIC RHPRESEMTATIOS}

Some results related to the modeling of deterministic TEG [3], [4] in max-algetra are presented in this section; for a general survey sec [1]- -44$]$.

To describe completely the behavior of a TEG, it suffices to record the seguences of its transition firing dates. We assume that transition are fired as soon as they can be fired. For a transition labeled $z$. we defne $z(k)$ as the date when transition $z$ is fired for the $k$ th time. For example, we consider two machines descrihed by the deterministic TEG of Fig. 1.

The firing of transition a means that a pan is given to the input stock (place Pl) to be manufactured by machine 1 . The firing of transition $\varepsilon_{M_{2}}$ denotes the loudine of a part in machine 1 when this oik is free (token in place P2). The holding time $d_{M 1}$ associated with place P3 indicates the warking time of machine 1. The firing of iransition $x_{M_{2}}$ meuns that machine 1 has just completed its work. Machine 2, which can manufacture a part in $d_{M r 2}$ seconds, works like machine 1. We state that

$$
\begin{aligned}
& x_{M_{1}}(k+1)=\max \left\{x_{M_{2}}(k), u(k+1)\right\} \\
& x_{M_{2}}(k+1)=d_{M 1}+x_{M_{1}}(k+1) .
\end{aligned}
$$

For $x M_{1}$ to be fired $k+1$ times, it is necessary that $u$ also be fired $k+1$ times, whereas $x_{M_{2}}$ needs only to be fired $k$ times since one token is already avaikble in place $P 2$. On the other hand, if $x_{M_{1}}$ protuces a token at a time $t$, this token will not be available before $i+d_{M 1}$ for use by $s_{M_{2}}$. The max operation neflects the behavior of the connections. Finally. the equality results from the assumption that transitions ane fired inmedistely when they can be fired.

Operations max and + are written in tuas-algebra as $(9$ and \&. respectively, to clearly underline the Inearity of a deteministic DES The elements of mux-atgebra are the real numbers and mirus infinity (denotad $e$ ). Zero is denoted $e$ to refer to the unity eicment of 9 More generally, the model we ecasider is described by the following linear equations in max-algebra [3]:

$$
\left\{\begin{aligned}
\Sigma_{M}(k+1) & =A \otimes \underline{x}_{M}(k) \oplus B \otimes \underline{u}(k+1) \\
\underline{y}_{M}(k) & =C \otimes \underline{x}_{M}(k) .
\end{aligned}\right.
$$

The model state (demoted $\left.x_{M}\right)$ is the $n$-vector $\left(x_{M}, \ldots x_{M}\right)^{2}$ The control (denoted $u$ ) is the $p$-vector $\left(u_{1} \ldots u_{p}\right)^{t}$, and the morlet output (denoted $\left.y_{y}\right)$ is the $q$-vector $\left(y M_{1} \quad \cdots y_{4}\right)^{4}$. The mutrices $A, B, C$ have appropriate dimensions with entries in $\sharp \cup\{c\}$. 


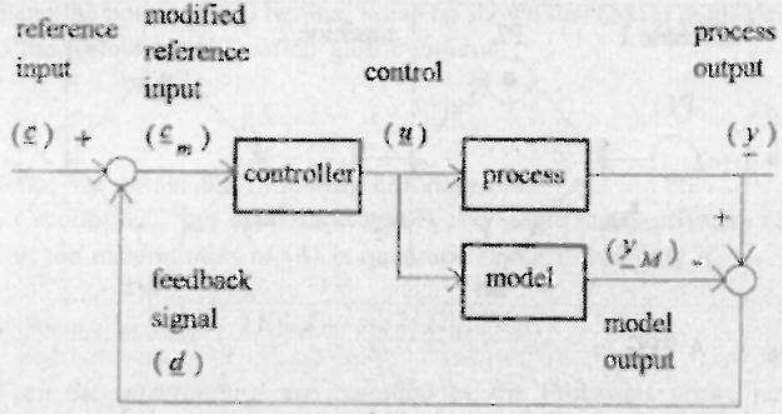

Fig. 2. Basic IMC stncture.

As an example, the deterministic TFG of Fig. 1 is described in max-algebra by

$$
\left\{\begin{array}{c}
\underline{x}_{M}(k+1)=\left[\begin{array}{ccc}
\varepsilon & e & \varepsilon \\
\varepsilon & d_{M} & \varepsilon \\
\varepsilon & d_{M 1} & d_{M 2}
\end{array}\right] \underline{x}_{M}(k) \\
\qquad\left[\begin{array}{c}
e \\
d_{M 1} \\
d_{M 1}
\end{array}\right] \otimes u(k+1) \\
y_{M}(k)=\left[\begin{array}{lll}
\varepsilon & d_{M 2}
\end{array}\right] \otimes r_{M}(k)
\end{array}\right.
$$

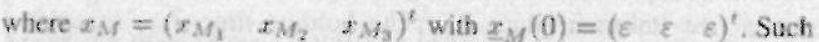
an initial model sate means tbat tokens of the initial marking are available immediately.

Henceforth, the sign $\otimes$ is omited as in usual linear algebra.

\section{BASTC INTERNAL MODEL CONTROI}

An open-loop control structure cannot guarantec to keep the process output close to the refercoce buput in case of mismatch between the process and its model (always present in practice). To solve this problem, the open-loop control structure is improved. The resulting control, whose structure is depicted in Fig. 2 , is called the basic IMC [5].

The modified reference input (denoted $c_{m}$ ) is equal to the reference input (denoted $c$ ) minus the difference between the process and motel outputs (denoted $y, y_{y}$, respectively). This difference (denoted $d$ and called the feedback signal) is due to an imperfect process modeling since the same control $\underline{u}$ is applicd to both process and model. Win such a control structure, the difference between the process output and the reference inpest is also equal to the one between the model output and the moditied reference input because $s_{m}=c-(y-1)$. Hence, to keep the process output as close as possible to the reference input, the model output muat behave as closely as posible to the modihed reference input which means that the controller is designed to be as close as possible to the inverse of the model (limitation is principally due to the necessary causality of the coutroller).

\section{BASIC INTER NAL MODRL CONTROE IN MAX-ALGERRA}

We try to control the firing dates of the discrete-event proces output $(y)$ by firing control $(v)$ at appsopriate instants. The reference input $(c)$ denctes the desired firing dates of the process ouput. The sequences of these fring dates are naturally nondecreasiag, moreover only the firing dafes of the teference input which occur before the instant when the control is computed are supposed avaisble to compute the controt

In the previous section, we have acen that the basic $I M C$ principle can be applied to a system when we can design a controller close to the imverse of its model. Hence, the use of this principle for DES raises the problem of nrodel inversion is max-aigebrs. Due to the feedhack signal design, we can note wha the IMC structure induces a ronlinearity in max-alucbu

Definition (Characteristic Number): For $h=1, \cdots$, . let vector $C$ be the kith row of matrix $C$, the characteristic number of the model oxtput $y_{M}$ whose behavior is described by $(1)$; if it exises, it is the smallest integer, denoted $\delta_{h}$, such that $C_{h} A^{\delta_{h}} B \neq \mathrm{E}$.

In the seque? to this paper, we assume that $\delta_{h}(h=1, \cdots, q)$ exist. Notation I: Let us define

$$
\Delta=\left[\begin{array}{c}
C_{1} A^{*} B \\
\vdots \\
C_{*} A^{\delta} \cdot B
\end{array}\right]
$$

ani

$$
I=\left[\begin{array}{c}
C_{1} A^{s_{2}+1} \\
\vdots \\
C_{2} A_{\gamma+1}
\end{array}\right]
$$

$\Delta$ and $F$ are $q \times p$ and $q \times$ is matrices, respectively: $\Delta h$ and $\Gamma_{h}$ are the hth row of matrices $\Delta$ and $\Gamma$, respectively.

Theorem: For $h=1, \cdots, \%$ we have

$$
g M_{h}\left(k+\delta_{h}+1\right)=\Gamma_{h} x_{M}(k) \oplus \Delta_{h} \underline{\underline{k}}(k+1) .
$$

Proof: By definition of $\delta h$, we have

$$
C_{h} B=\cdots=C_{h} A^{\delta_{h}-1} B=\varepsilon
$$

and

$$
\Delta \neq \underline{\underline{E}} .
$$

Hence, (1) implies that

$$
3 M_{k}(k+j)=G_{M} A^{j} \underline{x}_{M}(k)
$$

for

$$
j=0, \cdots, \delta \text {, }
$$

then

$$
\begin{aligned}
& y_{M}\left(k+\delta_{h}+1\right)=C_{h} A^{\delta_{3}} \underline{r}_{M}(k+1) \\
& =\Gamma_{n} \underline{x}_{M}(k) \oplus \Delta_{n \underline{u}}(k+1) \text {. }
\end{aligned}
$$

Remark 1: The first $\delta_{h}$ firing dates of the nortel output $y_{4}$ $(h=1, \cdots, q)$ only depend on inithal model state. We have

$$
H_{B}(j)=C_{h} A^{\prime} I_{M}(0)
$$

for

$$
j=0, \cdots, \delta_{h}
$$

According to (3) we see that control $u(k+1)$ can inlluence at the earliest the future model output $3 M_{n}\left(k+\delta_{h}+1\right)(h=1, \cdots, q)$. Hence, the design of a controller that is as close as possibte to the model inverse requires the knowledge of the desired future model output. i.e., the future modified reference input $c_{m_{\mathrm{A}}}\left(k+\delta_{h}+1\right)$ $(h=1, \cdots, q)$ when control $u(k+1)$ is calculated. But these values are unknown since they depend on both future reference imput $c_{h}\left(k+\delta_{h}+1\right)(h=1, \cdots, q)$ (not avallabie by assumption) and future fecdback signal $d_{s}\left(k+\delta_{h}+1\right)(h=1, \cdots, q)$, i.e., control $*(k+1)$. Hence, the controller we propose is split into fwo blocks called Prediction and hinversion (see Fig. 3).

Control $u(h+1)$ is calculated in the lnversion biock so that the future firing dates of the model oulput $y_{M_{A}}\left(k+\delta_{h}+1\right)(h=$ $1, \cdots, q)$ occur as close as possible to the ones defined by the predicted modified reference input. Let us note that in proctice the control components are not necesaraily calculated at the same instam 


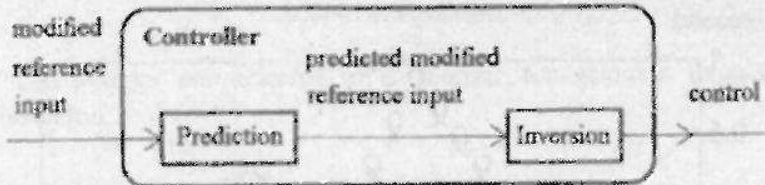

Fig. 3. Comtrolier.

because their occurrences can be differeat. Therefore, it is interesting to estimate the future modified reference input $c_{m_{\mathrm{s}}}\left(k+\partial_{2}+1\right)$ $(h=1, \cdots, q)$ in the Prediction block at each calculation of a control componerat $u_{2}(k+1)$ to have all the possible past values of the modified reference inpui. For $:=1, \ldots, p$, the values of the predicted modified reference input, which ase used when the control $u_{i}(k+1)$ is calculated, are denoted by

$$
\begin{aligned}
& \sum_{m}^{p}\left[n_{i}(k+1)\right]= \\
& \left\{c_{m_{1}}^{p\left[v_{1}(k+1)\right]}\left(k+\delta_{1}+1\right) \cdots c_{m_{q}}^{p_{k}\left(k_{i}(k+1)\right]}\left(k+\delta_{q}+1\right)\right\}
\end{aligned}
$$

Practically, we compute the $k+i t h$ firing date of the control component $u_{2}$ as soon as possible after the $k$ th firing date of the control component $u_{i}$.

The design of the Prediction block is not the aim of this note even if the prediction quality of the modified reference input is unportant for the success of the mode! inversion method. In the example we consider in Section $\mathrm{V}$, the next firing date of the reference input, used to calculate the control, is supposed to be known. Hence, only the feedhack signal (d) needs to be predicted. To simply predict correctly a constant behavior of the feedback signal, we use its latest-known value when the control is calculated. It is clear that such a prediction method is rubimentary

Let symbol $\odot$ refer to the multiplication of two matrices in which the mig-operation is used rather than the max-operation [3]: for al matrices $F, G$ with entries in $\Re \cup\{-\infty,+\infty\}$, we have

$$
(F \odot G)_{i j}=\min _{2}\left\{F_{i q}+G_{a j}\right\} \text {. }
$$

By convention, we have

$$
(-\infty) \otimes(+\infty)=(-\infty)
$$

but

$$
(-\infty) \cdot(+\infty)=1+\infty)
$$

Let us recall an important resuh due to the Residuation sheory [1]-[3]: Given a $q \times y$ matrix $\phi$ and a $q$-vector $\beta$ with entries in $\Re \cup\{-\infty,+\infty\}$, the greatest subsolution of $\Phi \underline{\alpha}=\beta$ exists and is given by $\alpha=\left(-\phi^{\prime}\right) \odot \underline{\beta}$.

Corollary 1: Let $\Delta^{\prime}$ be the ith column of the matrix $\Delta$

$$
\begin{gathered}
u_{i}(k+1)=\left(-\Delta^{i}\right)^{i} \cdot\left\{\underline{S}_{m}^{p\left(u_{i}(k+2)\right]} \oplus \Gamma \underline{\underline{x}}_{M}(k)\right\} \\
i=1, \ldots, p
\end{gathered}
$$

accurring at the latest so that $\Delta_{h} u_{i}(k+1)$ occurs at the latest date before:

$$
\Gamma_{h x_{M}}(k) \oplus c_{m_{k}}^{p[v(k+1)]}\left(k+\delta_{b}+1\right) \quad h=1, \cdots, q .
$$

The proof is a direct application of the Residuation theory.

Notation 2: For $h=1, \cdots, q$, ler us define $i_{\mathrm{s}}$ such that

$$
\Delta_{h}^{l} u_{l_{b}}(k+1)=\Delta_{h} u(k+1) \text {. }
$$

We can note that the possible value of $/ \mathrm{h}$ is not necessarily single, in this case any possible value can be considered.
Corvllary 2: Conerol $u(k+1)$ defined by $(5)$ occurs at the lates so that

$$
\begin{aligned}
& y_{M_{A}}\left(k+\delta_{h}+1\right)= \\
& \Gamma_{h \Sigma_{M}}(k) \oplus \Delta_{h}^{l_{h}}\left(\left(-\Delta^{l_{h}}\right)^{t} \odot\left\{c_{m}^{p\left[w_{h}(k+1)\right]} \oplus \Gamma_{x_{M}}(k)\right\}\right) \\
& h=1, \cdots, y
\end{aligned}
$$

More precisely, either $y M_{h}\left(k+\delta_{h}+1\right)$ accurs at the latest before $\operatorname{con}_{\mathrm{A}}\left(u_{h}(k+1)\right\}\left(k+b_{h}+1\right)$, i.e., at

$$
\Delta_{h}^{l_{k}}\left(\left(-\Delta^{l_{k}}\right)^{2} \odot\left\{c_{m}^{p\left[a_{k}(k+1)\right]} \oplus \Gamma_{x_{M}}(k)\right\}\right)
$$

or $y_{M_{h}}\left(k+\delta_{k}+1\right)$ occurs at the earliest after

$$
\Delta_{h}^{h}\left(\left(-\Delta^{l b}\right)^{t} \odot\left\{\operatorname{sm}^{p\left[u_{h}(k+1)\right\}} \oplus \Gamma_{x_{M}}(k)\right\}\right)
$$

i.e., at $\Gamma_{\text {has }}(k)$

Proof: From (3) and (6), and due to (5), we easily obtwin (7). If we suppose that

$$
\begin{aligned}
\Gamma_{h E_{M}}(k) & <\Delta_{h}^{h_{h}}\left(\left(-\Delta^{l_{k}}\right)^{t} \cdot\left\{\underline{s m}^{p\left[s_{h}(k+1)\right]} \oplus \Gamma_{x_{M}}(k)\right\}\right) \\
& =\Delta_{h}^{l_{h}} u_{h}(k+1)
\end{aligned}
$$

from $(7)$ it is clear that

$$
y M_{b}\left(k+\delta_{h}+1\right)=\Delta_{h}^{l_{h}} u_{t_{h}}(k+1)>\Gamma_{h} \underline{x}_{M}(k) .
$$

On the other hand, by usiag Corollary 1 we see that $\Delta_{h}^{l_{k}} u_{k}(k+1)$ occurs at the latest before $\left.\Gamma_{h} x_{M}(k) \oplus c_{m_{h}}^{p u_{2}}(k+1)\right]\left(k+\delta_{h}+1\right)$ which implies that $y M_{h}\left(k+\delta_{h}+1\right)$ cocurs at the latest before $c_{m h}^{p\left[w_{h}(k+1)\right]}\left(k+\delta_{b}+1\right)>\Gamma_{h} x_{M}(k)$. When

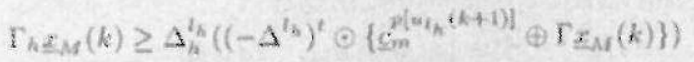

from (7) we see easily that $y_{M_{A}}\left(k+\delta_{h}+1\right)$ accurs at $\Gamma_{k} \underline{x}_{M}(k)$.

Remark 2: Control $u(k+1)$ defined by (5) needs the model state $x_{M}(k)$ which is easily avalahle in the IMC stracture.

Remark 3: In the particulas case where the modich is exactly known and the ralues of the predicted modified reference input are exact, we clearly have $c_{m h}[u(k+1)\}=c_{h}\left(k+\delta_{h}+1\right)(i=1, \cdots, p$, $h=1, \cdots, \varphi)$ which yields, according to Notation 2

$$
\left.c_{m h}^{p\left[i_{h}\right.}(k+1)\right]\left(k+\delta_{h}+1\right)=c_{h}\left(k+\delta_{h}+1\right) \quad h=1, \cdots, q \text {. }
$$

Hence, by using Corollary 2 we see that $y_{h}\left(k+\varepsilon_{h}+1\right)$ occurs at the latest before $c_{h}\left(k+\partial_{h}+1\right)$, i.e. at

$$
\left.\Delta_{h}^{i_{h}}\left(-\Delta^{h}\right)^{t} \odot\left\{\left[\begin{array}{c}
c_{1}\left(k+\delta_{1}+1\right) \\
\vdots \\
c_{q}\left(k+\delta_{q}+1\right)
\end{array}\right] \oplus \Gamma_{k}(k)\right\}\right)
$$

or at the earliest atter this wne, i.e. at $\Gamma_{h x}(k)$. Let us recull that the latter case is avoided when the adjoint systent can be used (see Section 1).

Remark 4: In single-input-single-output (SISO) case, Corollary 2 is simplified. The solution of $4 \alpha=\beta$ exists and is given by $\alpha=(-\$) \beta$. Thus control $u(k+1)$ accurs at the latest 50 thit $B M(k+\delta+1)=\Gamma_{I_{M}}(k) \oplus C_{m}^{[u(k+1)]}(k+\delta+1)$.

\section{ExaWHe}

We consider the rwo machines described in max-algebra by (2). Since $C B=d_{M 1} d_{M 2} \neq s$, the characteristic number $\delta$ exists and is equal to zero, thus $\Delta=C B$ and $I=C A$. From (5), we have

$$
u(k+1)=\{-\Delta)\left\{c_{m}^{p(k+1)\}}(k+1) \oplus \Gamma_{\mathcal{R}_{M}}(k)\right\}
$$




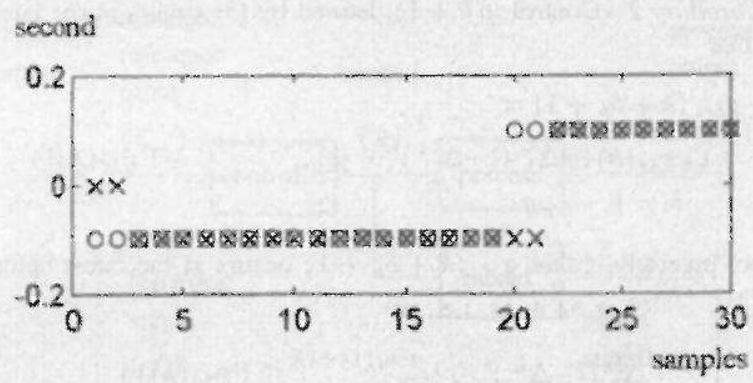

Fig. 4. Feedback signal $(d)$ o predicted feedhack signal $\left(d^{2}\right) \times$

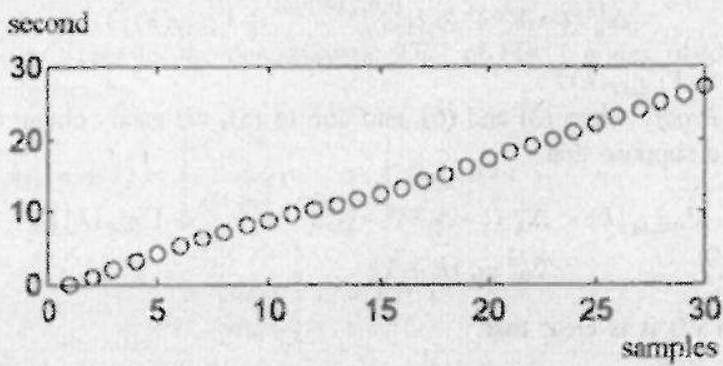

Fig. 5. Control $(u)$ o.

with

$$
(-\Delta)=\left(-d_{M 1}\right)\left(-d_{M 2}\right)
$$

and

$$
\Gamma=\left[\begin{array}{lll}
\varepsilon & d_{M 1} d_{M 2} & 2 d_{M 2}
\end{array}\right] .
$$

Hence, according to Remark 4 and owing to the IMC sructure (see Section (II), we have

$$
\begin{aligned}
y(k+1)-c(k+1)= & y_{M}(k+1)-c_{m}(k+1) \\
= & \left\{c_{m}^{j[(k+1)]}(k+1) \oplus \Gamma_{x_{M}}(k)\right\} \\
& -c_{m}(k+1) .
\end{aligned}
$$

In the simulation case, the reference input is defined by

$$
\begin{array}{ll}
c(k)=c(k-1)+1 & \text { for } k=1, \cdots, 9 \text { with } c(0)=0 \\
c(k)=c(k-1)+0.5 & \text { for } k=10, \cdots, 13 \text { and } \\
c(k)=c(k-1)+1 & \text { for } k \geq 14 .
\end{array}
$$

For convesience, only the manufacturing time of machine $2(d, / 2)$ is supposed to be imperfectly madeled: The value $d_{M 2}$ of the model. used for control design, is equal to $0.7 \mathrm{~s}$, while the true vatue is equal to $0.6 \mathrm{~s}$ during the first $19 \mathrm{samples}$ and to $0.8 \mathrm{~s}$ afterwards. The manufacturing time of machine $I\left(d_{M}\right)$ is exact and is equal to $0.2 \mathrm{~s}$

The reference input $c(k+1)$ is supposed to be known when contro $u(k+1)$ is computed fjust after $u(k)\}$, and hence ouly the feedback signal needs to be predicted. To predict it correctly when its behavior is constant, let $d^{p(a(k+1))}(k+1)=d(i)$, where $d(j)$ is the lasi firing date of the feedback sigual we dispose of when control $n(k+1)$ is computed.

Fig. 4 represenis the behaviors of leedback signal and its prediction. Initially, the model is supposed to be perfect: Let dp $(u(1)\}(1)=$ 0 . We can observe that the change of the feedback signal at sample 20 causes a wrong prediction at sumples 20 and 21 which is due to the poomess of the prediction method.

Fig. 5 represents the behuvior of control. Fig. 6 represents the behaviors of the error hetween the model outyut and the reference

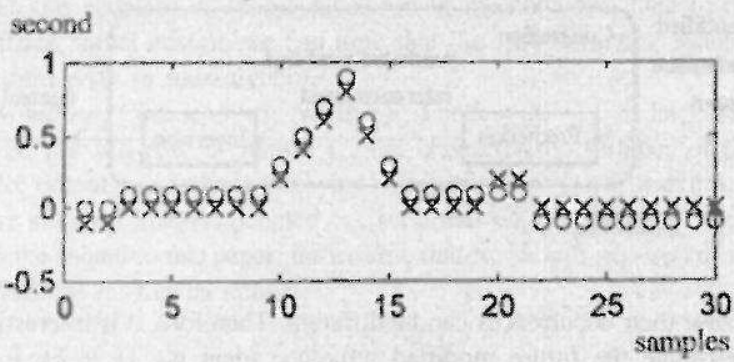

Fig. 6. Model outpul/reference input crnor (3y - c) 0 and process ont put/reference input error $(g-c) \times$.

input and of the error berween the process output and the reference input.

We observe in Fig. 5 that for samples 10-15. the control occurs as soon as possibie, i.e., $v(k)=(-\Delta) \Gamma x_{M}(k-1)$ for $k=10, \cdots, 15$ Two reasons induce this behavior:

- For samples 10-13, machine 2 cannot produce enough pans to satisfy the production rate desired by the reference input. Such a problem causes a divergence between the process ouput and the reference ingut at the same samples (sce Fig. 6).

- At samples 14 and 15 , the machines produce parts as soon as posible to zero the error between the process output and the reference input (see Fig. 6).

On the other hand, it can be seen in Fig. 6 that the process oupur is equal to the reference input in spite of a moufacturing time mismatch, when for the same sample the prediction of feedbach signa? is exact (see Fig. 4), and the control does not depend on the modet state.

\section{V1. Conclusto:}

We propose the use of IMC structure to try and take into accoun imperfect modeling for deterninistic DIS's. Such systems are described by linear equarions in max-algebra. In the basic IMC stroctare. the controller design raises the problem of model invension because the difference between the process output and the reference inpu is equat to the one between the model cusput and the controlle? input, i.e., the modified reference input. Due to the IMC structure we camot design the controller by using the aljoin system $[2$ Section X], [3. Section \$.6] which is ideally adapted to an openloop controi structure. The proposed controller is based on the future (predicted) modified reference input, and it is split into two blocks. called Prediction and Inversion. Our work concems only the Imversion block where Residuation theory plays an exoential role. Frring the model output as late as possible before desired rime instants is not always assured, the control firing dates oceur at the latest, so that for each model output:

- If the future firing date of the nodet output can occur before the one defined by the predicted motified reference inmut, then it occurs at the latest time before the one defined by the predicted modified reference input (oniy at in SISO case):

- Otherwise, the future firing date of the model output occur. at the earliest after the one defined by the predicted moditied reference input.

An important point is the robustress of the IMC. How can we guarantee stability in spitc of the important mismatch between the process and its model? A possible approach would consist in predicting the reference input separately from the feedback signal Hence, robustness would depend on the techrique used to predict the feedback signal. 


\section{ACKNOWLEDOMENT}

The authors are grateful to C. Ferrier for valuable linguistic discusaion.

\section{REHERFNCES}

[1] R. A. Cunisghame-Green, Minimuar Algebra (lecture Notes in Econom. ics and Mathematical Systems), vol. 166. New York: Springer-Verlag 1979.

[2i G. Cohen, P. Moller, 3. P. Quadrat, and M. Viut, "Adgebraic teols for the performance evaluation of discrefe eveat systems, "Proc. IEFE, vol. 77, no. 1. pp. 39-58, 1989

[3] F. Baccelli, (6. Cohca, G. J. Olsder, and J. P. Quadrat, Synchunization. and Lincarity: An Algebra for Discrete Event Systems, New York Wiley, 1992.

[4] T. Murata, "Petri nets: Propertics, anslysis, and apptications." Proc IEEE, voi. 77 , no. 4, pp. 541-580, 1989

[5] M. Morari and E. Zatixiou, Rotust Process Control. Englewerod Cliffs. Ni. Prentice-Hall, 1989

\section{Primciple of Proportional Damages in a Multiple Criteria LQR Problem}

Yuri B. Shiessel

Abstact - A multiple criteria linear quadratie regthator (LQR) prablea is considered. The principle of proportional damages is worked aut to miximize a norm of a difference between ideal and eptimal values of the vector-performance criteria, while the distribution of the losses (damages) of the performance criteria are under controt. a malihgle criteria LOR probkem solution vis principle of proportional damases is obtaincd as a linear forno of state variables and is invariast to the storms istich is manimired.

\section{INTRODUCTON}

The problem considered belongs to the domain of linear systems with guadratic performance crieriu. This is, for cxampic, a linearized flight control ptoblem [1]. The set of linearized equations of the motion of a rigid aircraft can be considered as three interacted suhsystems. Each subsystem describes pitch, yaw, and roll angle rates. Behavior of these angle rates can be chancterized by three quadratic performance criteria. These criteria can be minimized by means of corresponding choice of control functions: aileron, rudder, and clevator positions. Apparently, each control function affects all performance criteria. Cousezuently, an ar-vehicle fight controt is a typical multiple criteria control problem. Decospling control strategy, when initially subsystems are decoupled via decoupling control and then optimal control functions are identified in exch subsystem separately, is very popular. Unlike this traditionat approuch, we propose to employ interconnections between subsystems to improve the performance of each sabsystem. In this work we will look for the compromise minimization comtrol solution based on a procedure that calculates Pareto optianal solutions as the approximation to an ideal point $[2]-15]$. The distance between ideal and actual values

Manuscript received Jamuary 13, 1995

The author is with the Deparument of Elcctrical and Computer Engincering. Universtiy of Alabama, Huntsville, Al. 35999 USA

Publisher flem Identifier \$ 0018-9786(96) $02262-3$. of the vector-performance criteria is formed as some notm in the criteria space. This noru is usually mimimized [5] to synthesie the componised control strategy. This approach is attractive because of its mathematical and phvsical clarity. However, some disadvanages exist. First, it is not obvious how to specify the nom in the criteria space. The compromised solution shoukd depend on the form of this norm. Second, minimizing the nurn: we do not comerol the distribution of the differences between ideal and optimal (compromised) values of each criterion. It can lead w unaceptable compromised behaviot in some subsystems. The principle of proportional damages is worked cut [6]. [7] to minimize a norm of a difference between ideal and optimal values of the vector-criteria, white the distribution of the losses (damages) of each criterion is under control, A mulhple criteria linear quadratic. regulator $(L \mathrm{OR})$ moblem solution via princinle of proportional darnages is obtained as linear form of state variatles and is invariant to the norm which is minisuized.

\section{Promun FormulatoN}

Suppose a inear time-varying muli-input-multi-ouput system, consisting of $X$ interacting subsystems, is described by the systems of the differential equation.

$$
\begin{aligned}
i & =A(t) x(t)+B(t) u(t) \\
x(0) & =s_{0}
\end{aligned}
$$

where the entries of matrices $A(t) \in R^{n \times n}, B(t) \in R^{n \times m}$ are continuous functions of tine.

Each subsystem is characterized by the quadratic performance index (criterion)

$$
\begin{aligned}
J_{i}(x, u) & =\frac{1}{2} \int_{0}^{T}\left[x^{3}(t) Q_{i}(t) x(t)+u^{T}(t) R_{1}(t) x(t)\right] d t \\
\forall i & =1, N
\end{aligned}
$$

where time-dependem matrices $Q_{i}(t) \in R^{n \times n}, R_{i}(t) \in R^{m \times m}$ are positive semidetinite and positive definite, correspotndingly, while the performance of $(1)$ is characterized by the vector-eritersa

$$
J(x, u)=\left\{J_{1}(x, u), J_{2}(x, u), \cdots, J_{N}(x, u)\right\} .
$$

We wish to find an optimal feedback control law $u^{*}(t, x)$ such that (3) is minimized. We will think of (3) as minimized in the sense of the principle of proportional damaes [5], [0]

\section{PRINCIPLE OF PROPOKTIONAL DAMAGeS}

One of the widespread approaches to look for the compromise feedback control law $u^{*}(t, x)$ is based on a procedture that calculates Pareto optimat solutions as an approximation to an ideai poriat $j^{0}$ which is introduced in criteria space as follows $[2]-\{5]$ :

$$
j^{0}=\left\{J_{1}^{0}, J_{2}^{0}, \cdots, J_{N}^{0}\right\}
$$

shlucre

$$
\begin{aligned}
& J_{i}^{0}=J_{i}\left(x, u^{i}\right), J_{i}\left(x, u^{i}\right) \leq J,(x, u) \\
& \forall i=1, X .
\end{aligned}
$$

Very often a distance between (3) and ( 4 ) is represented as in the following norm:

$$
\left.\rho\left(J, J^{0}\right)=\left[\sum_{i=1}^{N} \mid J_{i}(x, u)-J_{i}^{\beta}\right]^{\beta}\right]^{1 / \rho}, p \in[1, \infty)
$$

